\title{
Focus on the neuroscience toolbox
}

\author{
We present a special issue on the neuroscience toolbox, highlighting recent technological advances, approaches and \\ collaborative initiatives that are enabling new avenues of research.
}

$\mathbf{T}$ he pace of technological development in neuroscience is accelerating, providing researchers with powerful new tools to drive their experiments and ask new questions. Keeping up with this wave of new technology can be challenging, and decisions about which tools to use and how best to use them can be difficult. Nature Neuroscience presents a special focus issue on the neuroscience toolbox aimed at helping researchers take full advantage of available techniques.

Researchers and funding agencies across the globe have recognized that the development of new tools and techniques is central to advancing basic neuroscience research and the treatment of disorders. On page 1118, we profile six worldwide initiatives involved in this effort: the Allen Institute for Brain Science, China Brain Project, the European Union's Human Brain Project, Israel Brain Technologies, Japan's Brain/MIND and the US BRAIN Initiative. The leaders of each of these initiatives have outlined their goals, achievements, and the challenges that lie ahead. Their goals are ambitious, yet it is clear that these focused efforts are enabling scientists to address major challenges ahead.

One such challenge is the vast degree of cellular heterogeneity in the nervous system. This has been parsed by single-cell gene expression profiling, enabling systematic classification and categorization of neuronal and non-neuronal cell types. Although expression profiling has been possible for decades, recent advances in microfluidics and sequencing have made it possible to implement more easily and less expensively. In their Review on page 1131, Rajeshwar Awatramani and colleagues provide a guide to the use of single-cell transcriptomics, examples of its successful deployment, and implications of its use for understanding cellular specialization in the nervous system. This piece marks the first of a series of technical reviews that we plan to publish in the future aimed at promoting data quality and reproducibility in neuroscience.

Analyses at the single-cell and population level have also been advanced by genetically encoded indicators of neuronal activity. Spikes, dendritic and synaptic potentials, vesicle fusion and neurotransmitter release events can all be tracked optically. On page 1142, Michael Lin and Mark Schnitzer offer an analysis of genetically encoded indicators of neuronal activity. From $\mathrm{pH}$ and neurotransmitters to voltage and calcium, the authors discuss the various designs, their characteristics and behavior, strengths and weaknesses, and the way they can and should be used by experimenters to answer different types of questions.

The increased number and type of optical sensors and actuators available has propelled our understanding of brain circuitry at cellular and subcellular resolution. But circuits are often distributed over large volumes of brain matter, and imaging them in action at a speed sufficient to track action potentials and synaptic activity is a challenge. In their Review on page 1154, Na Ji, Jeremy Freeman and Spencer Smith discuss current approaches to volumetric imaging of the brain, focusing on the instrumental challenges associated with the use of optical systems. They provide an overview of optimal sampling strategies for rapid imaging in three dimensions and the computational and analytical approaches necessary to handle the size and complexity of the resulting datasets.

Simultaneous recordings of activity from hundreds to thousands of neurons-optically or electrically_-require massively parallel recordings and sophisticated algorithms, both automatic and manually-curated, operating with sufficient accuracy and selectivity to return high yield single units. In our second technical review, on page 1165, Kenneth Harris and colleagues discuss state-of-the-art techniques for inferring single spikes from electrophysiology and two-photon calcium imaging. They focus on practices that are meant to ensure data quality and discuss problems in interpretation when the data quality is compromised.

Our understanding of how discrete populations of neurons are connected and function together has been advanced by the Human Connectome Project, which has endeavored to produce maps of human brain connections. Its systematic study of a large cohort of subjects with multiple neuroimaging modalities has led to a wealth of knowledge on how to optimally perform human brain imaging. On page 1175, Matthew Glasser, David Van Essen and their colleagues share technical insights and provide a series of integrated guidelines organized around seven tenets on how to approach data acquisition, analysis and sharing in human neuroimaging laboratories. This is a valuable reference on how to access and analyze the Human Connectome Project data, or adopt and adapt the project's approach for one's own research. A comprehensive set of Supplementary Notes is available to allow the reader to delve deeper into the technical details of the approach.

One of the core motivating factors in the push to develop new techniques for interrogating brain function is to understand brain disorders. Beyond the tools themselves, there need to be suitable, etiologically relevant animal models of disease. Guoping Feng and colleagues present a Perspective on page 1123 on nonhuman primates as a tool for understanding psychiatric disease. They discuss the rationale for developing transgenic primate models and new strategies for manipulating the primate genome, articulating the challenges, both technical and ethical, that lie ahead in this complex yet necessary endeavor.

In assembling this focus, our aims are to affirm our commitment towards publishing the latest technical innovations, improving methods of data analysis and reporting, and to highlight the advantages of collaborative efforts. These technical advancements and initiatives are providing fundamental insights into the workings of the nervous system. We look forward to the breakthroughs and conceptual insights rendered possible by these techniques and approaches, as well as to the next generations of tools that will emerge in coming years. 